INPLASY

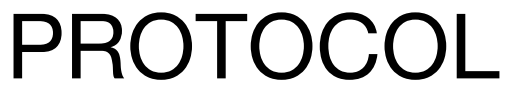

To cite: Liu et al. Effectiveness of Xiaoyao San in treatment of Diabetes combined with Depression: A Systematic Review and Meta-analysis. Inplasy protocol 2021100075. doi:

10.37766/inplasy2021.10.0075

Received: 20 October 2021

Published: 20 October 2021

Corresponding author:

Xiaomin Liu

xiaomin3452021@163.com

Author Affiliation:

Guangdong Metabolic Diseases

Research Center of Integrated

Chinese and Western Medicine;

Guangdong Pharmaceutical

University; The First Affiliated

Hospital of Guangdong

Pharmaceutical University

Support: None.

Review Stage at time of this submission: Preliminary

searches.

Conflicts of interest:

None declared.

\section{Effectiveness of Xiaoyao San in treatment of Diabetes combined with Depression: A Systematic Review and Meta-analysis}

Liu, XM1; Meng, ZZ2; Liu, XY3; Piao, SH4.

Review question / Objective: This study is to systematically evaluate the efficacy and safety of Xiaoyao San combined with or without conventional treatment for diabetes combined with depression was evaluated by meta-analysis.

Condition being studied: Diabetes combined with depression is globally prevalent. The condition affects quality of life, glucose control, nonadherence to treatment, cost of living, mortality, and life expectancy. It has been shown that people with diabetes combined with depression have higher mortality rates than individuals with diabetes exclusively. Xiaoyao San is used for the prevention and treatment of multiple-system diseases such as psychiatric disorders, neurological diseases, digestive system diseases, gynecologic diseases, and endocrine diseases.

INPLASY registration number: This protocol was registered with the International Platform of Registered Systematic Review and Meta-Analysis Protocols (INPLASY) on 20 October 2021 and was last updated on 20 October 2021 (registration number INPLASY2021100075).

\section{INTRODUCTION}

Review question / Objective: This study is to systematically evaluate the efficacy and safety of Xiaoyao San combined with or without conventional treatment for diabetes combined with depression was evaluated by meta-analysis.

Condition being studied: Diabetes combined with depression is globally prevalent. The condition affects quality of 
life, glucose control, nonadherence to treatment, cost of living, mortality, and life expectancy. It has been shown that people with diabetes combined with depression have higher mortality rates than individuals with diabetes exclusively. Xiaoyao San is used for the prevention and treatment of multiple-system diseases such as psychiatric disorders, neurological diseases, digestive system diseases, gynecologic diseases, and endocrine diseases.

\section{METHODS}

Search strategy: We will conduct literature searches from the following electronic databases: PUBMED, Web of Science, Cochrane Library, Embase, SinoMed, China National Knowledge Infrastructure (CNKI), China Science and Technology Journal Database, and Wanfang Database. The search strategy will include medical subject terms (MeSH) and keywords related to the treatment of diabetes combined with depression, and the search time will be set to the time of establishment of the database until October 2021. And by cross-checking the reference lists of all the included main studies and the list of related systematic reviews. Grey literature, Chinese Clinical Trial Registry, and Clinical Trials will also be searched as supplements.

Participant or population: Diabetes combined with depression patients diagnosed in accordance with the guidelines will be included, without limitation of age, gender and race.

Intervention: Xiaoyao San combined with or without conventional treatment.

\section{Comparator: Placebo or Conventional treatment.}

Study designs to be included: Trials that compared Xiao Yao San or modified Xiao Yao San as the active intervention in the treatment group versus placebo or conventional medicines will be included. Randomized controlled trials (RCTs) will be included.
Eligibility criteria: (1)Diabetes combined with depression patients diagnosed in accordance with the guidelines will be included, without limitation of age, gender and race;(2)Interventions: The experimental group was administered Xiaoyao San combined with or without conventional treatment. (3) Comparison: the control group was treated with Placebo or Conventional treatment.; (4)Study design: randomized controlled trials will be included; (5) Outcome indicators: total effective rate, Fasting blood glucose(FBG), HbAlc, Hamilton Depression Scale (HAMD).

Information sources: The two researchers independently searched and extracted pieces of literature from PUBMED, Web of Science, Cochrane Library, Embase, SinoMed, China National Knowledge Infrastructure (CNKI), China Science and Technology Journal Database, and Wanfang Database after selecting keywords and matching free words. The search period is from the database establishment to October 2021, regardless of language. The search strategy will take the form of medical subject headings (MeSH) and keywords and optimize the search strategy according to the characteristics of different databases. Keywords include: "Xiaoyao San", "xiao yao", "Diabetes", "depression", "randomized controlled trial", "clinical efficacy", etc.

Main outcome(s): The main outcomes will include total effective rate, the levels of blood glucose (FBG, HbAlc), HAMD.

Additional outcome(s): Additional outcomes will include but are not limited to clinical syndrome integral, quality of life, sleep disorders, adverse events, etc.

Data management: Two authors independently conducted research selection and data extraction. Any disagreements will be resolved through discussion until a consensus is reached or a third author is consulted. The extracted information includes several aspects: the overall characteristics of the study, the general characteristics of the subjects, the 
detailed information of the methodological information, the detailed information of the treatment plan, the detailed information of the control intervention measures (including conventional treatment drugs), the research results (including clinical and Laboratory parameters) and adverse effects.

Quality assessment / Risk of bias analysis: Two authors will independently assess the risk of bias in included researches. The quality of included trials will be assessed by using the risk of bias tool according to the Cochrane Handbook of Systematic Reviews of Interventions' to address the following five criteria: random sequence generation, allocation concealment, blinding of participants and personnel, blinding of outcome assessment, incomplete outcome data, and selective reporting.

Strategy of data synthesis: The results of the included studies will be descriptively synthesized, including participant characteristics and intervention details. We will calculate the effect size by weighted average/standard average deviation (for continuous results) and $95 \% \mathrm{Cl}$ odds ratio (OR) and $95 \% \mathrm{Cl}$ (for binary results). The $\mathrm{X}^{2}$ test will be used to evaluate the heterogeneity and $I^{2}$ will be used to assess the inconsistency across studies. Values of $I^{2}$ ranged from 0 to $100 \%\left(I^{2}<40 \%\right.$, might not be important; $30 \%<I^{2}<60 \%$, moderate heterogeneity; $50 \%<\mathrm{I}^{2}<90 \%$, substantial heterogeneity; $75 \%<\mathrm{I}^{2}<100 \%$, considerable heterogeneity). . Use RevMan5.3 provided by the Cochrane Collaboration Network for meta-analysis. Potential publication bias will be assessed graphically with funnel plot.If there is clinical and methodological heterogeneity, a subgroup analysis will be performed to explore the source of the heterogeneity in the study.

Subgroup analysis: Subgroup analyses will be conducted according to interventions in trial group and in control group.

Sensitivity analysis: We plan to conduct sensitivity analysis by deleting individual trials one by one and repeating metaanalysis to explore the reasons for the heterogeneity between studies.

Language: English.

Country(ies) involved: China.

Keywords: Xiaoyao San; diabetes; depression; Meta-analysis.

Contributions of each author:

Author 1 - Xiaomin Liu.

Author 2 - Zhenzhen Meng.

Author 3 - Xinyu Liu.

Author 4 - Shenghua Piao. 